\title{
Hadronic molecules for charmed and bottom baryons near thresholds
}

\author{
Yasuhiro Yamaguchi ${ }^{1}$, Shunsuke Ohkoda ${ }^{1}$, Shigehiro Yasui ${ }^{2}$, and Atsushi Hosaka ${ }^{1}$ \\ ${ }^{1}$ Research Center for Nuclear Physics (RCNP), \\ Osaka University, Ibaraki, Osaka, 567-0047, Japan and \\ ${ }^{2}$ KEK Theory Center, Institute of Particle and Nuclear Studies, \\ High Energy Accelerator Research Organization, \\ 1-1, Oho, Ibaraki, 305-0801, Japan
}

(Dated: September 10, 2018)

\begin{abstract}
We study hadronic molecules formed by a heavy meson and a nucleon, $D N$ and $D^{*} N(\bar{B} N$ and $\left.\bar{B}^{*} N\right)$ systems. Respecting the heavy quark symmetry and chiral symmetry, we consider the $D N-D^{*} N\left(\bar{B} N-\bar{B}^{*} N\right)$ mixing induced by the one boson exchange potential including the tensor force. We find many bound and resonant states with $J^{P}=1 / 2^{ \pm}, 3 / 2^{ \pm}, 5 / 2^{ \pm}$and $7 / 2^{-}$in isospin singlet channels, while only a few resonant states with $J^{P}=1 / 2^{-}$in isospin triplet channels. The analysis of $D N$ and $D^{*} N\left(\bar{B} N\right.$ and $\left.\bar{B}^{*} N\right)$ molecules will be useful to study mass spectra of excited charmed (bottom) baryons with large angular momenta, when their masses are close to the $D N$ and $D^{*} N\left(\bar{B} N\right.$ and $\left.\bar{B}^{*} N\right)$ thresholds.
\end{abstract}

PACS numbers: 12.39.Hg, 14.20.Lq, 14.20.Mr, 14.20.Pt 


\section{INTRODUCTION}

Recently, many exotic hadrons observed in experiments are attracting our interest. They are expected to have unexpected structures, because their properties, such as quantum numbers, masses, and decay patterns, cannot be explained by the ordinary picture of hadrons, baryons as $q q q$ and mesons as $q \bar{q}$. As one of new aspects of these structures, the possibility of multiquarks or hadronic molecules have been extensively discussed. It is considered that, particularly near thresholds, hadronic molecules such as loosely bound or resonant states of meson-meson or meson-baryon emerge as one of hadronic configurations realized in nature. As typical candidates, the meson-meson molecules for $X(3872)$ and $Z_{b}$ have been studied in the charm and bottom quark regions $[1-8]$.

The hadronic molecules as bound or resonant states formed by $D$ or $D^{*}$ meson $\left(\bar{B}\right.$ or $\bar{B}^{*}$ meson) and a nucleon $(N)$ are also interesting. Since the $D N$ and $D^{*} N\left(\bar{B} N\right.$ and $\left.\bar{B}^{*} N\right)$ molecules contain ordinary flavor structures of three quarks, they are intimately related to the ordinary heavy baryons, $\Lambda_{\mathrm{c}}, \Sigma_{\mathrm{c}}, \Sigma_{\mathrm{c}}^{*}$ and their excited states for the charm sector, and $\Lambda_{\mathrm{b}}, \Sigma_{\mathrm{b}}, \Sigma_{\mathrm{b}}^{*}$ and their excited states for the bottom sector [9]. These excited states have been studied in the quark model extensively [11, 12]. However, near the $D N$ and $D^{*} N(\bar{B} N$ and $\left.\bar{B}^{*} N\right)$ thresholds, it is expected that the wave functions of the quark model states have a large overlap with the component of $D N$ and $D^{*} N\left(\bar{B} N\right.$ and $\left.\bar{B}^{*} N\right)$, and the properties of such baryon states are strongly affected by $D N$ and $D^{*} N\left(\bar{B} N\right.$ and $\left.\bar{B}^{*} N\right)$ states. There would be even such states that are dominated by the molecular components.

In the $D N$ and $D^{*} N\left(\bar{B} N\right.$ and $\left.\bar{B}^{*} N\right)$ systems, there are two important symmetries; the heavy quark symmetry and chiral symmetry. The heavy quark symmetry manifests in mass degeneracy of heavy pseudoscalar mesons $P=D, \bar{B}$ and vector mesons $P^{*}=D^{*}, \bar{B}^{*}$ in the heavy quark mass limit [13, 14]. Indeed, the mass splitting between a $D(\bar{B})$ meson and a $D^{*}\left(\bar{B}^{*}\right)$ meson is small; $140 \mathrm{MeV}$ for $D$ and $D^{*}$ mesons and $46 \mathrm{MeV}$ for $\bar{B}$ and $\bar{B}^{*}$ mesons. The small mass splittings in the heavy flavor sectors should be compared with the large mass splittings, $\sim 400 \mathrm{MeV}$ for $\bar{K}$ and $\bar{K}^{*}$ mesons, and $\sim 600 \mathrm{MeV}$ for $\pi$ and $\rho$ mesons, in the light flavor sectors. Because of this, the heavy quark symmetry introduces the mixing of $P N$ and $P^{*} N$ states, where both $P$ and $P^{*}$ mesons are considered on the same footing as fundamental degrees of freedom in the dynamics [15]. Then, physical states are given as a superposition of $P N$ and $P^{*} N$ states. For convenience, in the following, we denote $P^{(*)}$ 
to stand for $P$ or $P^{*}$. Thanks to the mixing of states in $P^{(*)} N$ systems, a long range force between a $P^{(*)}$ meson and a nucleon $N$ is supplied by one pion exchange with the same coupling strengths for $P P^{*} \pi$ and $P^{*} P^{*} \pi$ vertices [16 18].

It is interesting that the pion exchange can exist in $P^{(*)} N$ systems. As a matter of fact, it is known that the pion exchange is important for the binding of atomic nuclei [19]. There the tensor force of the pion exchange that mixes channels with different angular momenta, i.e. $L$ and $L \pm 2$, yields a strong attraction to generate a rich structure of bound as well as resonant states. Because the pion exchange is a long range force, it becomes more significant for states with larger orbital angular momenta. In fact, it was shown that, in the nucleonnucleon scattering, the phase shifts in the channels with large orbital angular momenta are reproduced almost only by the pion exchange potential [20, 21]. The importance of the pion exchange was also discussed in $N \bar{N}$ systems. It was investigated that the properties of bound and resonant states in $N \bar{N}$ systems are dramatically changed, if the tensor force is switched off [22, 23]. In our previous studies, it was also shown that the pion exchange potential played a significant role for hadronic molecules such as $\bar{D} N$ and $B N$ baryons [16 18], $D \bar{D}$ $(B \bar{B})$ mesons (e.g. $Z_{b}$ meson) [24] and $D D(B B)$ mesons [25].

In reality, however, the hadronic molecules of $P^{(*)} N$ do not necessarily correspond to the observed states, because there should be couplings not only to three quark states but also to other meson-baryon states such as $\pi \Lambda_{\mathrm{c}}, \pi \Sigma_{\mathrm{c}}$ and $\pi \Sigma_{\mathrm{c}}^{*}\left(\pi \Lambda_{\mathrm{b}}, \pi \Sigma_{\mathrm{b}}\right.$ and $\left.\pi \Sigma_{\mathrm{b}}^{*}\right)$, and so on. However, we may expect that such couplings are small for $D N$ and $\bar{B} N$ baryons near the thresholds. The reasons are that the wave functions of hadronic molecules are spatially large as compared to the conventional three quark states, and that the transitions, e.g. from $D N$ $(\bar{B} N)$ to $\pi \Sigma_{\mathrm{c}}\left(\pi \Sigma_{\mathrm{b}}\right)$, are suppressed by a heavy quark exchange. From those points of view, in the present discussion, we focus on the role of the $D^{(*)} N$ and $\bar{B}^{(*)} N$ sectors and study the bound or resonant states generated by $D^{(*)} N$ and $\bar{B}^{(*)} N$. Recently, there have been several studies of baryon states as $D^{(*)} N\left(\bar{B}^{(*)} N\right)$ [26 36$]$ and $\bar{D}^{(*)} \Sigma_{\mathrm{c}}\left(B^{(*)} \Sigma_{\mathrm{b}}\right)$ [36 38$]$ molecules. The present study is different from them in that we focus on the states near the thresholds and with a large angular momentum.

This paper is organized as follows. In Sec. II, we briefly describe the interaction between a $D^{(*)}$ or $\bar{B}^{(*)}$ meson and a nucleon. In Sec. III, we solve the coupled channel Schrödinger equations numerically for bound and resonant states. We discuss the results of the $D^{(*)} N$ and $\bar{B}^{(*)} N$ molecule in Sec. IV, and summarize the present work in the final section. 


\section{INTERACTIONS}

Following our previous studies [16 18], we consider $\pi, \rho$ and $\omega$ exchange potentials between $P^{(*)}$ and $N$. The interaction Lagrangians for heavy meson vertices are given by the heavy quark symmetry and chiral symmetry [39, 40],

$$
\begin{aligned}
\mathcal{L}_{\pi H H} & =i g_{\pi} \operatorname{Tr}\left[H_{b} \gamma_{\mu} \gamma_{5} A_{b a}^{\mu} \bar{H}_{a}\right], \\
\mathcal{L}_{v H H} & =-i \beta \operatorname{Tr}\left[H_{b} v^{\mu}\left(\rho_{\mu}\right)_{b a} \bar{H}_{a}\right]+i \lambda \operatorname{Tr}\left[H_{b} \sigma^{\mu \nu} F_{\mu \nu}(\rho)_{b a} \bar{H}_{a}\right],
\end{aligned}
$$

where the subscripts $\pi$ and $v$ are for the pion and vector ( $\rho$ and $\omega$ ) meson interactions. The heavy meson field $H$ is defined by $H_{a}=\frac{1+p}{2}\left[P_{a \mu}^{*} \gamma^{\mu}-P_{a} \gamma_{5}\right]$ with the four-velocity $v^{\mu}$ of a heavy meson, where the subscript $a$ is for light flavors, $u, d$. The conjugate field is given by $\bar{H}_{a}=\gamma_{0} H_{a}^{\dagger} \gamma_{0}$. The axial current of light flavors is written by $A^{\mu}=\frac{1}{2}\left(\xi^{\dagger} \partial^{\mu} \xi-\xi \partial^{\mu} \xi^{\dagger}\right)$ with $\xi=\exp \left(i \hat{\pi} / f_{\pi}\right)$ and the pion decay constant $f_{\pi}=132 \mathrm{MeV}$. The pion field is defined by

$$
\hat{\pi}=\left(\begin{array}{cc}
\frac{\pi^{0}}{\sqrt{2}} & \pi^{+} \\
\pi^{-} & -\frac{\pi^{0}}{\sqrt{2}}
\end{array}\right),
$$

and the vector meson field by

$$
\begin{aligned}
& \rho^{\mu}=i \frac{g_{V}}{\sqrt{2}} \hat{\rho}_{\mu} \\
& \hat{\rho}_{\mu}=\left(\begin{array}{cc}
\frac{\rho^{0}}{\sqrt{2}} & \rho^{+} \\
\rho^{-} & -\frac{\rho^{0}}{\sqrt{2}}
\end{array}\right)_{\mu},
\end{aligned}
$$

where $g_{V} \simeq 5.8$ corresponds to the gauge coupling constant from hidden local symmetry [41]. The vector meson field tensor is written by $F_{\mu \nu}(\rho)=\partial_{\mu} \rho_{\nu}-\partial_{\nu} \rho_{\mu}+\left[\rho_{\mu}, \rho_{\nu}\right]$. The coupling constants $g_{\pi}, \beta$ and $\lambda$ are summarized in Table I. These coupling constants are essentially the same as those given in Refs. [17, 18], except for the signs of $g_{\pi}, \beta$ and $\lambda$ for vertices of $\pi$ and $\omega$, which are reversed due to G-parity transformation between $D^{(*)}\left(\bar{B}^{(*)}\right)$ and $\bar{D}^{(*)}$ $\left(B^{(*)}\right)$. In Eqs. (11) and (2), we consider the static approximation $v^{\mu}=(1, \overrightarrow{0})$.

For vertices of $\pi, \rho$ and $\omega$ mesons to a nucleon, we employ the Bonn model [42] as

$$
\begin{aligned}
\mathcal{L}_{\pi N N} & =\sqrt{2} i g_{\pi N N} \bar{N} \gamma_{5} \hat{\pi} N \\
\mathcal{L}_{v N N} & =\sqrt{2} g_{v N N}\left[\bar{N} \gamma_{\mu} \hat{\rho}^{\mu} N+\frac{\kappa}{2 m_{N}} \bar{N} \sigma_{\mu \nu} \partial^{\nu} \hat{\rho}^{\mu} N\right]
\end{aligned}
$$


TABLE I. Masses and coupling constants of mesons $\alpha=\pi, \rho, \omega$ in Refs. [17, 18].

\begin{tabular}{lcccccc}
\hline Meson & $m_{\alpha}$ & $g_{\pi}$ & $\beta$ & $\lambda\left[\mathrm{GeV}^{-1}\right]$ & $g_{\alpha N N}^{2} / 4 \pi$ & $\kappa$ \\
\hline$\pi$ & 137.27 & -0.59 & - & - & 13.6 & - \\
$\rho$ & 769.9 & - & 0.9 & 0.59 & 0.84 & 6.1 \\
$\omega$ & 781.94 & - & -0.9 & -0.59 & 20.0 & 0.0 \\
\hline
\end{tabular}

where $N=(p, n)^{T}$ is the nucleon field. The coupling constants for nucleons are summarized in Table 1 .

When we consider the $\pi, \rho$ and $\omega$ exchange potentials between $P^{(*)}$ meson and nucleon, we take into account internal structure of hadrons by introducing the monopole-type form factors at each vertex;

$$
F_{\alpha}(\Lambda, \vec{q})=\frac{\Lambda^{2}-m_{\alpha}^{2}}{\Lambda^{2}+\left|\vec{q}^{2}\right|}
$$

with the mass $m_{\alpha}$, three momentum $\vec{q}$ of the incoming meson $\alpha(=\pi, \rho, \omega)$, and the cutoff parameter $\Lambda$. Here, we introduce two cutoff parameters for a heavy meson $\left(D^{(*)}\right.$ and $\left.\bar{B}^{(*)}\right)$ and a nucleon. The cutoff parameter $\Lambda_{N}$ for the nucleon is determined to reproduce the properties (the binding energy, the scattering length and effective range) of the deuteron. The cutoff parameter $\Lambda_{P}$ for heavy meson $P^{(*)}=D^{(*)}, \bar{B}^{(*)}$ is determined by the ratios of matter radii of the heavy meson and nucleon where the ratio is obtained by a quark model calculation [16 18]. Their numerical values are summarized in Table II]

In the present study, we employ two types of potential: the $\pi$ exchange and the $\pi \rho \omega$ exchanges. By comparing the results from them, we show the importance of the one pion exchange potential.

TABLE II. Cutoff parameters of a nucleon $\left(\Lambda_{N}\right)$ and heavy mesons $\left(\Lambda_{D}\right.$ for $D^{(*)}$ meson and $\Lambda_{B}$ for $\bar{B}^{(*)}$ meson) as employed in Ref. [17, 18].

\begin{tabular}{lccc}
\hline Potential & $\Lambda_{N}[\mathrm{MeV}]$ & $\Lambda_{D}[\mathrm{MeV}]$ & $\Lambda_{B}[\mathrm{MeV}]$ \\
\hline$\pi$ & 830 & 1121 & 1070 \\
$\pi \rho \omega$ & 846 & 1142 & 1091 \\
\hline
\end{tabular}


TABLE III. Various coupled channels for a given quantum number $J^{P}$.

\begin{tabular}{|c|c|}
\hline$J^{P}$ & channels \\
\hline $1 / 2^{-}$ & $P N\left({ }^{2} S_{1 / 2}\right) \quad P^{*} N\left({ }^{2} S_{1 / 2}\right) \quad P^{*} N\left({ }^{4} D_{1 / 2}\right)$ \\
\hline $1 / 2^{+}$ & $P N\left({ }^{2} P_{1 / 2}\right) \quad P^{*} N\left({ }^{2} P_{1 / 2}\right) \quad P^{*} N\left({ }^{4} P_{1 / 2}\right)$ \\
\hline $3 / 2^{-}$ & $P N\left({ }^{2} D_{3 / 2}\right) \quad P^{*} N\left({ }^{4} S_{3 / 2}\right) \quad P^{*} N\left({ }^{4} D_{3 / 2}\right) \quad P^{*} N\left({ }^{2} D_{3 / 2}\right)$ \\
\hline $3 / 2^{+}$ & $P N\left({ }^{2} P_{3 / 2}\right) \quad P^{*} N\left({ }^{2} P_{3 / 2}\right) \quad P^{*} N\left({ }^{4} P_{3 / 2}\right) \quad P^{*} N\left({ }^{4} F_{3 / 2}\right)$ \\
\hline $5 / 2^{-}$ & $P N\left({ }^{2} D_{5 / 2}\right) P^{*} N\left({ }^{2} D_{5 / 2}\right) P^{*} N\left({ }^{4} D_{5 / 2}\right) P^{*} N\left({ }^{4} G_{5 / 2}\right)$ \\
\hline $5 / 2^{+}$ & $P N\left({ }^{2} F_{5 / 2}\right) \quad P^{*} N\left({ }^{4} P_{5 / 2}\right) \quad P^{*} N\left({ }^{2} F_{5 / 2}\right) \quad P^{*} N\left({ }^{4} F_{5 / 2}\right)$ \\
\hline $7 / 2^{-}$ & $P N\left({ }^{2} G_{7 / 2}\right) \quad P^{*} N\left({ }^{4} D_{7 / 2}\right) \quad P^{*} N\left({ }^{2} G_{7 / 2}\right) \quad P^{*} N\left({ }^{4} G_{7 / 2}\right)$ \\
\hline $7 / 2^{+}$ & $P N\left({ }^{2} F_{7 / 2}\right) \quad P^{*} N\left({ }^{2} F_{7 / 2}\right) \quad P^{*} N\left({ }^{4} F_{7 / 2}\right) \quad P^{*} N\left({ }^{4} H_{7 / 2}\right)$ \\
\hline
\end{tabular}

\section{NUMERICAL RESULTS}

We consider the states with $J^{P}=1 / 2^{ \pm}, 3 / 2^{ \pm}, 5 / 2^{ \pm}$and $7 / 2^{ \pm}$(total angular momentum $J$ and parity $P$ ) with isospin $I=0$ and 1 . Various states with $J^{P}$ are expanded by coupled channels of ${ }^{2 S+1} L_{J}$ (spin $S$ and orbital angular momentum $L$ ) as summarized in Table. III, The meson exchange potentials among various channels and kinetic terms are summarized in Appendix A Using the hamiltonian composed of the kinetic and potential terms, we solve the coupled-channel Schrödinger equations for $P N$ and $P^{*} N$ channels numerically.

\section{A. Isospin singlet $(I=0)$}

We present the results for the isosinglet state $(I=0)$. The energies of bound and resonant states are summarized in Table IV] and also presented in Figs. 1 and 2, The results are shown for the $\pi$ and $\pi \rho \omega$ potentials, and the corresponding energy levels are connected by arrows in Figs. 1 and 2. The numerical values are measured from the $D N$ and $\bar{B} N$ thresholds, respectively.

First we discuss the states with $J^{P}=1 / 2^{ \pm}$and $3 / 2^{-}$, and then those with $J^{P}=3 / 2^{+}$, $5 / 2^{ \pm}$and $7 / 2^{ \pm}$. In fact, it will turn out that the results in those two categories have different behaviors. 


\section{1. $J^{P}=1 / 2^{ \pm}$and $3 / 2^{-}$}

For $\left(I, J^{P}\right)=\left(0,1 / 2^{-}\right)$, we find bound states in both of $D^{(*)} N$ and $\bar{B}^{(*)} N$. For $D^{(*)} N$, the binding energies are $-14.4 \mathrm{MeV}$ for the $\pi$ potential and $-82.5 \mathrm{MeV}$ for the $\pi \rho \omega$ potential. The relative radii are $1.51 \mathrm{fm}$ and $0.86 \mathrm{fm}$, respectively. As expected, for a larger binding energy the system becomes smaller. The tensor force from the $\pi$ exchange which is the main driving force of the $D N-D^{*} N$ mixing plays an important role to form the bound states. In fact, we have verified that neither bound nor resonant state exists when the tensor force from the $\pi$ exchange is switched off and the $D N-D^{*} N$ mixing is small. For $J^{P}=1 / 2^{-}$, the results in the $\pi$ and $\pi \rho \omega$ potentials are very different as indicated in Figs. 1 and 2 , Since both $\rho$ and $\omega$ exchanges are attractive for $D^{(*)} N\left(\bar{B}^{(*)} N\right)$ system, the vector meson exchanges contribute to form the deeply bound state of the binding energy around $80 \mathrm{MeV}$. This contrasts with the previous result for the $\bar{D}^{(*)} N$ and $B^{(*)} N$ systems of truly exotic channels, where the $\rho$ and $\omega$ exchanges play a minor role due to the cancellation of these two potentials [17, 18].

We estimate the mixing ratio of various channels in the bound states as summarized in Table $\mathrm{V}$, We observe that, for $J^{P}=1 / 2^{-}$states, the most dominant component is $D N\left({ }^{2} S_{1 / 2}\right)$ with a fraction $71.8 \%$. The second dominant component is $D^{*} N\left({ }^{4} D_{1 / 2}\right)$ with a fraction 20.4 \%. Therefore, the tensor force which mixes the $S$-wave in $D N\left({ }^{2} S_{1 / 2}\right)$ and the $D$-wave in $D^{*} N\left({ }^{4} D_{1 / 2}\right)$ is important to provide a strong attraction.

For $\bar{B}^{(*)} N$ of $\left(I, J^{P}\right)=\left(0,1 / 2^{-}\right)$, we also obtain bound states. The binding energies are $-57.8 \mathrm{MeV}$ for the $\pi$ potential and $-145.9 \mathrm{MeV}$ for the $\pi \rho \omega$ potential, and the relative radii are $0.99 \mathrm{fm}$ and $0.76 \mathrm{fm}$, respectively. Again the results of the two potentials are very different with the same reason for the $D^{(*)} N$ system. As compared to the $D^{(*)} N$ system, the binding energy in the $\bar{B}^{(*)} N$ system is much larger, because heavier particles suppress kinetic energy. For the mixing ratios, we also find a similar tendency as we discussed for the $D^{(*)} N$ system; $56.1 \%$ for $\bar{B} N\left({ }^{2} S_{1 / 2}\right), 13.3 \%$ for $\bar{B}^{*} N\left({ }^{2} S_{1 / 2}\right)$ and $30.6 \%$ for $\bar{B}^{*} N\left({ }^{4} D_{1 / 2}\right)$.

Let us move to $\left(I, J^{P}\right)=\left(0,1 / 2^{+}\right)$state. For $D^{(*)} N$, we find one resonance near the $D N$ threshold when the $\pi$ potential is used. The resonance energy is $1.4 \mathrm{MeV}$ and the half decay width is $0.2 \mathrm{MeV}$ as shown in Table IV In the scattering state, we define the resonance energy $E_{\text {re }}$ by an inflection point of the phase shift [43], and the decay width $\Gamma$ is given by $\Gamma=2 /(d \delta / d E)_{E=E_{\mathrm{re}}}$. When the $\pi \rho \omega$ potential is used, we find a bound state with a binding 
TABLE IV. Properties of bound and resonant states of $D^{(*)} N$ and $\bar{B}^{(*)} N$ systems. The energies $E$ are either pure real for bound states or complex for resonant states. The complex energies for resonances are written as $E_{\mathrm{re}}-i \Gamma / 2$ where $E_{\mathrm{re}}$ is the resonance energy and $\Gamma / 2$ is the half-width. The binding and resonance energies are measured from the lowest threshold $(D N$ and $\bar{B} N)$. Root mean square radii are shown only for bound states.

\begin{tabular}{|c|c|c|c|c|c|}
\hline \multirow{2}{*}{$I\left(J^{P}\right)$} & \multirow{2}{*}{ Potential } & \multicolumn{2}{|c|}{$D N$} & \multicolumn{2}{|c|}{$\bar{B} N$} \\
\hline & & $E[\mathrm{MeV}]$ & $\left\langle r^{2}\right\rangle^{1 / 2}[\mathrm{fm}]$ & $E[\mathrm{MeV}]$ & $\left\langle r^{2}\right\rangle^{1 / 2}[\mathrm{fm}]$ \\
\hline \multirow{2}{*}{$0\left(1 / 2^{-}\right)$} & $\pi$ & -14.4 & 1.51 & -57.8 & 0.99 \\
\hline & $\pi \rho \omega$ & -82.5 & 0.86 & -145.9 & 0.76 \\
\hline \multirow{2}{*}{$0\left(1 / 2^{+}\right)$} & $\pi$ & $1.4-i 0.2$ & - & -83.8 & 0.92 \\
\hline & $\pi \rho \omega$ & -81.5 & 0.85 & -185.0 & 0.75 \\
\hline \multirow{2}{*}{$0\left(3 / 2^{-}\right)$} & $\pi$ & $63.5-i 7.9$ & - & -38.7 & 0.99 \\
\hline & $\pi \rho \omega$ & -13.7 & 0.89 & -127.8 & 0.76 \\
\hline \multirow{2}{*}{$0\left(3 / 2^{+}\right)$} & $\pi$ & $23.8-i 118.1$ & - & $12.9-i 15.5$ & - \\
\hline & $\pi \rho \omega$ & $26.0-i 44.2$ & - & -2.6 & 1.81 \\
\hline \multirow{2}{*}{$0\left(5 / 2^{-}\right)$} & $\pi$ & $153.6-i 671.9$ & - & $63.7-i 177.6$ & - \\
\hline & $\pi \rho \omega$ & $160.0-i 375.4$ & - & $71.3-i 102.8$ & - \\
\hline \multirow{2}{*}{$0\left(5 / 2^{+}\right)$} & $\pi$ & $160.8-i 3.1$ & - & $46.0-i 1.1$ & - \\
\hline & $\pi \rho \omega$ & $137.0-i 7.6$ & - & $20.0-i 0.2$ & - \\
\hline \multirow{2}{*}{$0\left(7 / 2^{-}\right)$} & $\pi$ & $217.7-i 182.4$ & - & $85.6-i 74.5$ & - \\
\hline & $\pi \rho \omega$ & $220.8-i 109.1$ & - & $87.5-i 46.7$ & \\
\hline \multirow{2}{*}{$0\left(7 / 2^{+}\right)$} & $\pi$ & no & - & no & - \\
\hline & $\pi \rho \omega$ & no & - & no & - \\
\hline \multirow{2}{*}{$1\left(1 / 2^{-}\right)$} & $\pi$ & no & - & no & - \\
\hline & $\pi \rho \omega$ & $147.2-i 105.5$ & - & $50.7-i 75.5$ & - \\
\hline
\end{tabular}

energy $-81.5 \mathrm{MeV}$ and with a relative radius $0.85 \mathrm{fm}$. The mixing ratios of the bound state are $38.8 \%$ for $D N\left({ }^{2} P_{1 / 2}\right), 6.0 \%$ for $D^{*} N\left({ }^{2} P_{1 / 2}\right)$ and $55.2 \%$ for $D^{*} N\left({ }^{4} P_{1 / 2}\right)$, as shown in Table. V] Interestingly, $D^{*} N\left({ }^{4} P_{1 / 2}\right)$ is the most dominant channel, although the mass of 


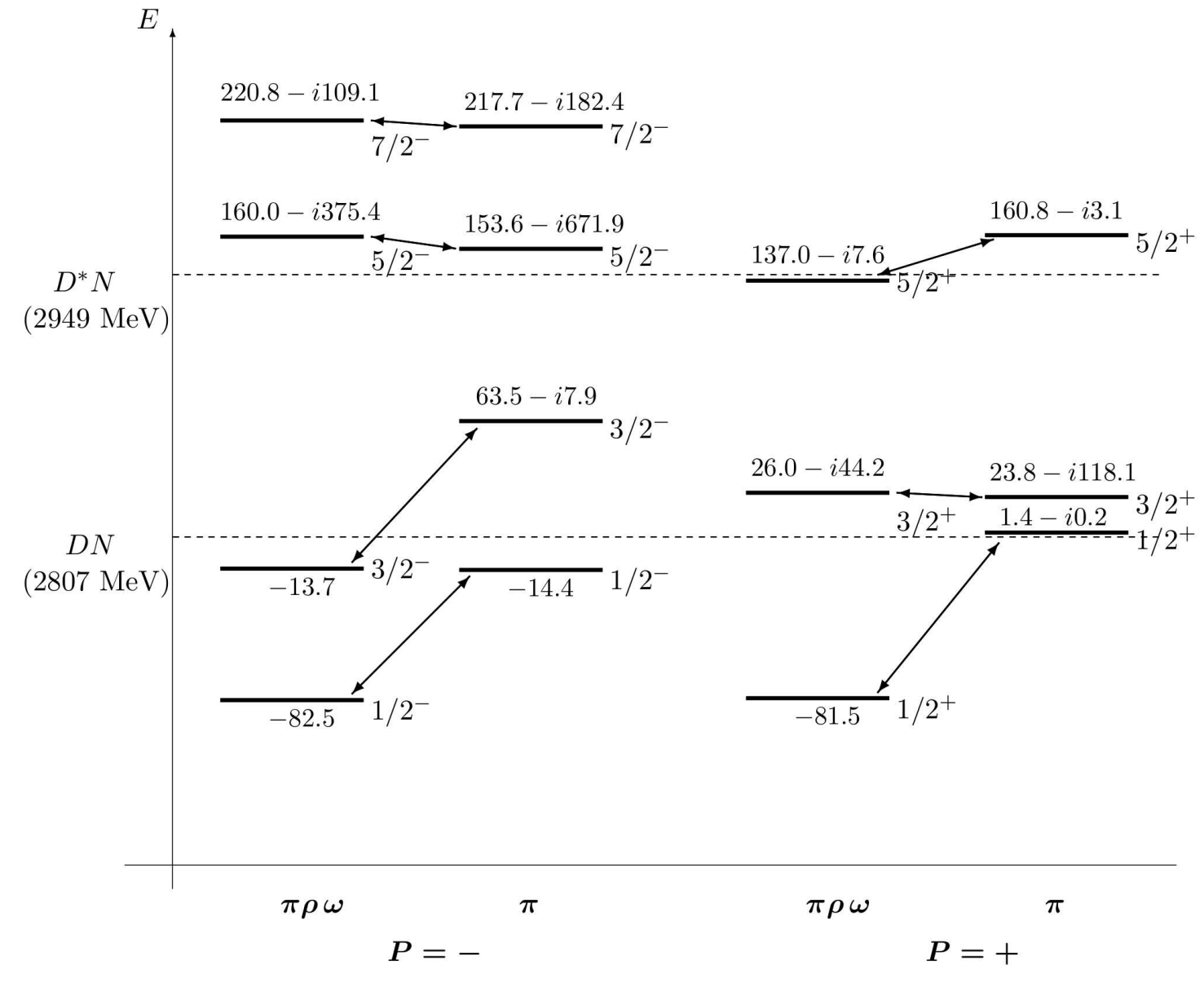

FIG. 1. Energies of bound and resonant states of $D^{(*)} N$ for $I=0$ with positive parity $(P=+)$ and negative parity $(P=-)$. The energies are measured from the $D N$ threshold. The results for $\pi$ and $\pi \rho \omega$ potentials are shown, where corresponding states are connected by arrows. The binding energies are given as real negative value, and the resonance energies $E_{\mathrm{re}}$ and decay widths $\Gamma$ are given as $E_{\mathrm{re}}-i \Gamma / 2$.

$D^{*} N$ is heavier than the mass of $D N$. This is because the attraction of tensor force is the strongest for $D^{*} N\left({ }^{4} P_{1 / 2}\right)$ channel.

For $\bar{B}^{(*)} N$ of $\left(I, J^{P}\right)=\left(0,1 / 2^{+}\right)$, we find bound states for both cases when the $\pi$ and $\pi \rho \omega$ potentials are used. The binding energies are $-83.8 \mathrm{MeV}$ for the $\pi$ potential and -185.0 $\mathrm{MeV}$ for the $\pi \rho \omega$ potential, and the relative radii are $0.92 \mathrm{fm}$ and $0.75 \mathrm{fm}$, respectively. For the $\pi \rho \omega$ potential, the mixing ratios of the bound state are $28.4 \%$ for $\bar{B} N\left({ }^{2} P_{1 / 2}\right), 7.7$ $\%$ for $\bar{B}^{*} N\left({ }^{2} P_{1 / 2}\right)$ and $63.9 \%$ for $\bar{B}^{*} N\left({ }^{4} P_{1 / 2}\right)$. In this case again, $\bar{B}^{*} N\left({ }^{4} P_{1 / 2}\right)$ is the most 


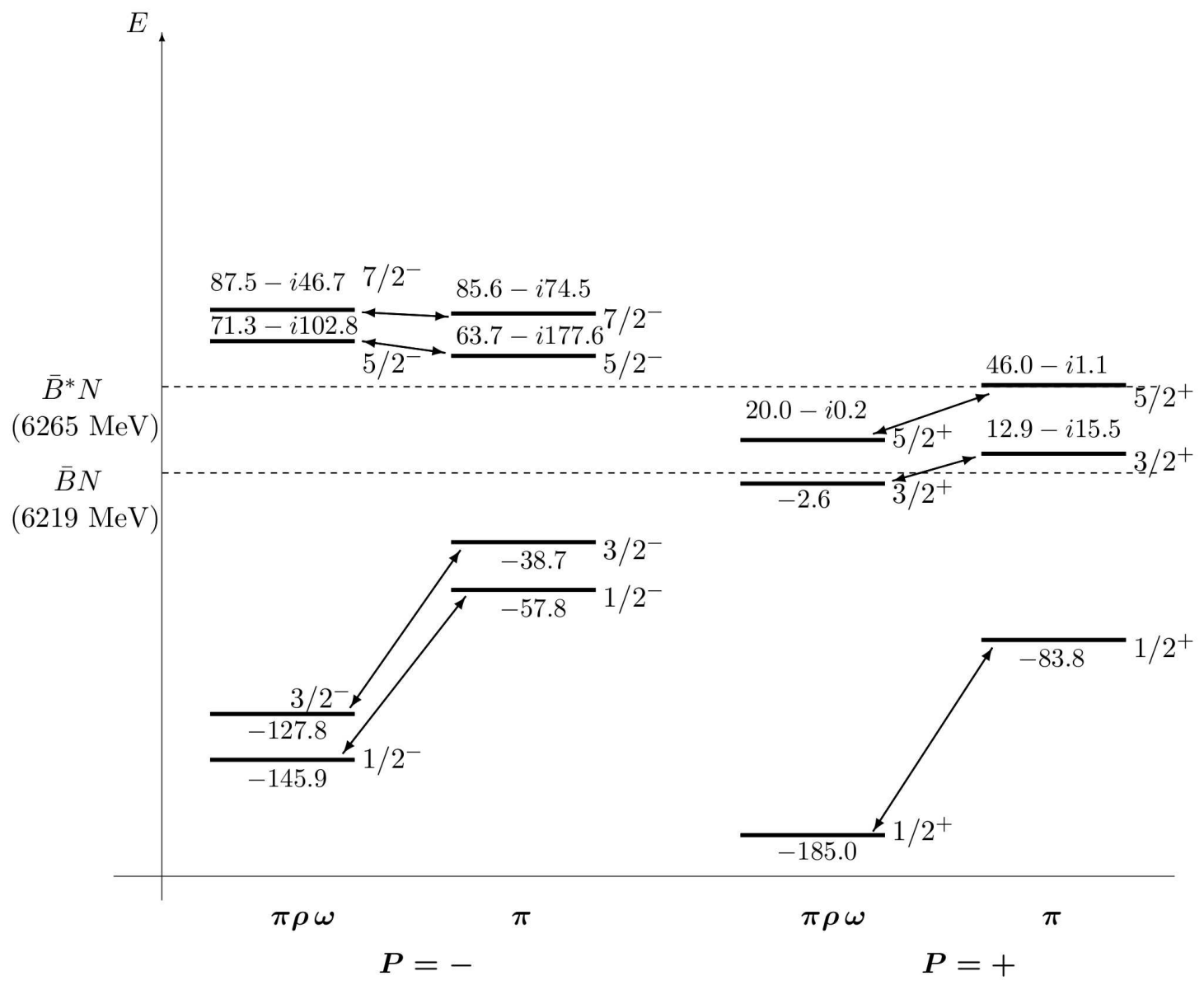

FIG. 2. Energies of bound and resonant states of $\bar{B}^{(*)} N$ for $I=0$ with positive parity $(P=+)$ and negative parity $(P=-)$. The energies are measured from the $\bar{B} N$ threshold. The same convention is used as Fig 1,

dominant component regardless of its heavy mass.

For $\left(I, J^{P}\right)=\left(0,3 / 2^{-}\right)$, we find a resonant $D N$ state for the $\pi$ potential with the resonance energy $63.5 \mathrm{MeV}$ and the half decay width $7.9 \mathrm{MeV}$ as shown in Table IV. In contrast, for the $\pi \rho \omega$ potential, we find a bound $D N$ state with the binding energy -13.7 $\mathrm{MeV}$ and the relative radius $0.89 \mathrm{fm}$. For the bound state, the mixing ratios are $19.8 \%$ for $D N\left({ }^{2} D_{3 / 2}\right), 62.8 \%$ for $D^{*} N\left({ }^{4} S_{3 / 2}\right), 14.2 \%$ for $D^{*} N\left({ }^{4} D_{3 / 2}\right)$ and $3.2 \%$ for $D^{*} N\left({ }^{2} D_{3 / 2}\right)$. Thus, $D^{*} N\left({ }^{4} S_{3 / 2}\right)$ is the dominant channel although its mass is heavier than the mass of $D N\left({ }^{2} D_{3 / 2}\right)$; once again a large attraction due to the tensor force is provided.

For $\bar{B}^{(*)} N$ state of $\left(I, J^{P}\right)=\left(0,3 / 2^{-}\right)$, we obtain bound states for both cases the $\pi$ 
TABLE V. Mixing ratio of each channel in the bound $D^{(*)} N$ and $\bar{B}^{(*)} N$ states for $J^{P}=1 / 2^{ \pm}$and $3 / 2^{ \pm}$with $I=0$ when the $\pi \rho \omega$ potential is employed.

\begin{tabular}{|c|c|c|c|c|c|c|c|c|c|}
\hline $1 / 2^{-}$ & $P N\left({ }^{2} S_{1 / 2}\right)$ & $P^{*} N\left({ }^{2} S_{1 / 2}\right)$ & $P^{*} N\left({ }^{4} D_{1 / 2}\right)$ & - & $1 / 2^{+}$ & $P N\left({ }^{2} P_{1 / 2}\right)$ & $P^{*} N\left({ }^{2} P_{1 / 2}\right)$ & $P^{*} N\left({ }^{4} P_{1 / 2}\right)$ & - \\
\hline$D N$ & $71.8 \%$ & $7.8 \%$ & $20.4 \%$ & - & $D N$ & $38.8 \%$ & $6.0 \%$ & $55.2 \%$ & - \\
\hline $\bar{B} N$ & $56.1 \%$ & $13.3 \%$ & $30.6 \%$ & - & $\bar{B} N$ & $28.4 \%$ & $7.7 \%$ & $63.9 \%$ & - \\
\hline $3 / 2^{-}$ & $P N\left({ }^{2} D_{3 / 2}\right)$ & $P^{*} N\left({ }^{4} S_{3 / 2}\right)$ & $P^{*} N\left({ }^{4} D_{3 / 2}\right)$ & $P^{*} N\left({ }^{2} D_{3 / 2}\right)$ & $3 / 2^{+}$ & $P N\left({ }^{2} P_{3 / 2}\right)$ & $P^{*} N\left({ }^{2} P_{3 / 2}\right)$ & $P^{*} N\left({ }^{4} P_{3 / 2}\right)$ & $P^{*} N\left({ }^{4} F_{3 / 2}\right)$ \\
\hline$D N$ & $19.8 \%$ & $62.8 \%$ & $14.2 \%$ & $3.2 \%$ & $D N$ & - & - & - & - \\
\hline $\bar{B} N$ & $14.6 \%$ & $64.7 \%$ & $16.7 \%$ & $4.0 \%$ & $\bar{B} N$ & $71.2 \%$ & $6.7 \%$ & $7.5 \%$ & $14.6 \%$ \\
\hline
\end{tabular}

and $\pi \rho \omega$ potentials are used. The binding energies are $-38.7 \mathrm{MeV}$ for the $\pi$ potential and $-127.8 \mathrm{MeV}$ for the $\pi \rho \omega$ potential with relative radii $0.99 \mathrm{fm}$ and $0.76 \mathrm{fm}$, respectively.

\section{2. $J^{P}=3 / 2^{+}, 5 / 2^{ \pm}$and $7 / 2^{ \pm}$}

For $\left(I, J^{P}\right)=\left(0,3 / 2^{+}\right)$, we find resonant states above the threshold. For $D^{(*)} N$, the resonance energies are $23.8 \mathrm{MeV}$ for the $\pi$ potential and $26.0 \mathrm{MeV}$ for the $\pi \rho \omega$ potential. The half decay widths are $118.1 \mathrm{MeV}$ and 44.2 MeV, respectively. As compared to the cases of $1 / 2^{ \pm}$and $3 / 2^{-}$, the results of the $\pi$ and $\pi \rho \omega$ potentials are not very much different, as indicated in Fig. 1. Since the wave functions are extended due to larger orbital angular momenta of $P$-wave $(L=1)$ and $F$-wave $(L=3)$ for $J^{P}=3 / 2^{+}$, the long range potential of the $\pi$ exchange dominates, while the short range potentials from $\rho$ and $\omega$ exchanges are suppressed. For $\bar{B}^{(*)} N$, when the $\pi$ potential is used, we find a resonant state whose resonance energy is $12.9 \mathrm{MeV}$ and half decay width is $15.5 \mathrm{MeV}$, while when the $\pi \rho \omega$ potential is used, we find a loosely bound state of a binding energy $-2.6 \mathrm{MeV}$ and a relative radius $1.81 \mathrm{fm}$. The mixing ratios are $71.2 \%$ for $\bar{B} N\left({ }^{2} P_{3 / 2}\right), 6.7 \%$ for $\bar{B}^{*} N\left({ }^{2} P_{3 / 2}\right), 7.5 \%$ for $\bar{B}^{*} N\left({ }^{4} P_{3 / 2}\right)$ and $14.6 \%$ for $\bar{B}^{*} N\left({ }^{4} F_{3 / 2}\right)$. The most dominant component is $\bar{B} N\left({ }^{2} P_{3 / 2}\right)$, and the second dominant one is $\bar{B}^{*} N\left({ }^{4} F_{3 / 2}\right)$.

For $\left(I, J^{P}\right)=\left(0,5 / 2^{-}\right)$, we obtain resonant states for both cases when the $\pi$ and $\pi \rho \omega$ potentials are used. For $D^{(*)} N$, the resonance energies are $153.6 \mathrm{MeV}$ for the $\pi$ potential and 
160.0 $\mathrm{MeV}$ for the $\pi \rho \omega$ potential, which are above the $D^{*} N$ threshold. The corresponding half decay widths are $671.9 \mathrm{MeV}$ and $375.4 \mathrm{MeV}$, respectively. The difference between the results of the $\pi$ and $\pi \rho \omega$ potentials is once again small, due to the same reason as before with large angular momenta. For $\bar{B}^{(*)} N$ state, we also find resonant states above the $\bar{B}^{*} N$ threshold. The resonance positions are $63.7 \mathrm{MeV}$ for the $\pi$ potential and $71.3 \mathrm{MeV}$ for the $\pi \rho \omega$ potential. The corresponding half decay widths are $177.6 \mathrm{MeV}$ and $102.8 \mathrm{MeV}$, respectively.

For $\left(I, J^{P}\right)=\left(0,5 / 2^{+}\right)$, we find resonant states with narrow widths for both cases when the $\pi$ and $\pi \rho \omega$ potentials are used. For $D^{(*)} N$, when the $\pi$ potential is used, the resonance energy is $160.8 \mathrm{MeV}$ and the half decay width $\Gamma / 2=3.1 \mathrm{MeV}$. When the $\pi \rho \omega$ potential is used, the resonance energy is $137.0 \mathrm{MeV}$ and the half decay width $\Gamma / 2=7.6 \mathrm{MeV}$. For $\bar{B}^{(*)} N$, we also find resonances whose energies are $46.0 \mathrm{MeV}$ for the $\pi$ potential and 20.0 $\mathrm{MeV}$ for the $\pi \rho \omega$ potential, with the corresponding half decay widths $1.1 \mathrm{MeV}$ and $0.2 \mathrm{MeV}$, respectively. Again, the results for the $\pi$ and $\pi \rho \omega$ potentials are similar.

For $\left(I, J^{P}\right)=\left(0,7 / 2^{-}\right)$, we obtain resonances above the $D^{*} N$ and $\bar{B}^{*} N$ thresholds. For $D^{(*)} N$, there exist resonances at $217.7 \mathrm{MeV}$ for the $\pi$ potential and at $220.8 \mathrm{MeV}$ for the $\pi \rho \omega$ potential, with half decay widths $182.4 \mathrm{MeV}$ and $109.1 \mathrm{MeV}$, respectively. For $\bar{B}^{(*)} N$, there also exist resonances whose energies are $85.6 \mathrm{MeV}$ for the $\pi$ potential and $87.5 \mathrm{MeV}$ for the $\pi \rho \omega$ potential, with half decay widths $74.5 \mathrm{MeV}$ and $46.7 \mathrm{MeV}$, respectively.

Finally, for $\left(I, J^{P}\right)=\left(0,7 / 2^{+}\right)$, we find no bound nor resonant state.

\section{B. Isospin triplet $(I=1)$}

Let us move to the states of isospin triplet $(I=1)$. We summarize the results for $D^{(*)} N$ and for $\bar{B}^{(*)} N$ in Table IV and show the energy levels in Fig. 3. As a result, we find resonant states only for $J^{P}=1 / 2^{-}$when the $\pi \rho \omega$ potential is employed. For $D^{(*)} N$, the resonance energy is $147.2 \mathrm{MeV}$ and the half decay width is $105.5 \mathrm{MeV}$. For $\bar{B}^{(*)} N$ state, we obtain the resonance whose energy is $50.7 \mathrm{MeV}$ and half width $75.5 \mathrm{MeV}$. The reason that there are not many states in isospin triplet channel can be understood as follows; as compared to the isosinglet channel, the attractive force in the isotriplet channel is weak due to small isospin factor; $\vec{\tau}_{P} \cdot \vec{\tau}_{N}=-3$ for isosinglet and $\vec{\tau}_{P} \cdot \vec{\tau}_{N}=1$ for isotriplet. 

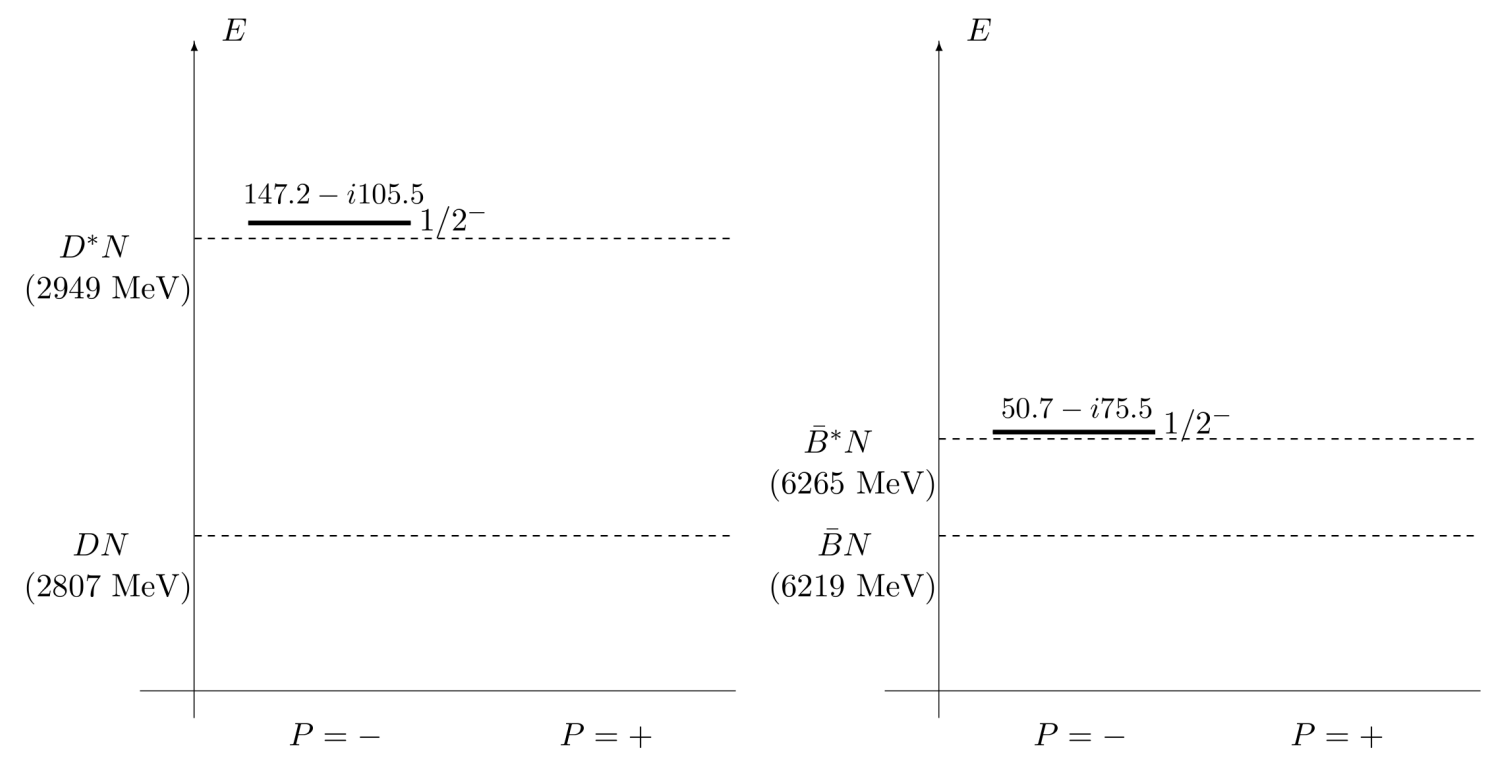

FIG. 3. Energies of bound and resonant states of $D^{(*)} N$ and $\bar{B}^{(*)} N$ for $I=1$ when the $\pi \rho \omega$ potential is used. The same convention is used as Fig 1.

\section{DISCUSSION}

\section{A. Contribution from short range interactions}

By solving coupled channel Schrödinger equations for $P N$ and $P^{*} N$ channels, we find many bound and resonant states for $I=0$ and few resonances for $I=1$. For these states, the tensor force of the $\pi$ exchange potential plays a significant role to produce them. When we ignore the $P^{*} N$ channels and solve the Schrödinger equation only with $P N$ channels, we find no bound state nor resonance. Thus, the $P N-P^{*} N$ mixing and the $\pi$ exchange potential play an important role to generate a rich structure of molecular states.

The importance of the $\pi$ exchange potential stands out more in the bottom sector. The small mass difference between $\bar{B}$ and $\bar{B}^{*}$ mesons helps to yield the strong attraction because it induces the strong $\bar{B} N-\bar{B}^{*} N$ mixing with the tensor force. Furthermore, the $\bar{B}^{(*)} N$ has heavier reduced mass and hence the kinetic term is suppressed. For these reasons, the binding energies of $\bar{B}^{(*)} N$ states are larger than these of $D^{(*)} N$ states.

When we compare the results of the $\pi$ potential and of the $\pi \rho \omega$ potential, they are quite different for $J^{P}=1 / 2^{ \pm}, 3 / 2^{-}$with $I=0$, where the $\rho$ and $\omega$ exchange potentials become 
important to produce attraction. For $D^{(*)} N$ and $\bar{B}^{(*)} N$ states, both $\rho$ and $\omega$ exchange potentials are attractive, and hence they increase the binding energy significantly. On the other hand, for $J^{P}=3 / 2^{+}, 5 / 2^{ \pm}$and $7 / 2^{-}$, the results for the $\pi \rho \omega$ potential are similar to those for the $\pi$ potential. For large $J$ states, the $\pi$ exchange potential plays a dominant role to generate bound and resonant states, while the $\rho$ and $\omega$ exchanges play only a minor one. This is attributed to the fact that these states have large orbital angular momenta. If relevant channels include large orbital angular momenta, the wave functions tend to extend spatially, and the long range force, namely the $\pi$ exchange potential, becomes important while the short range force is suppressed.

\section{B. Emergent hadronic molecules}

In the present study, we discuss the molecular structure formed by the $P^{(*)} N$ bound and resonant states. However, the hadronic molecular picture is not applicable to deeply bound states with small radii. For such states, the constituent hadrons, namely $D^{(*)}\left(\bar{B}^{(*)}\right)$ meson and nucleon, overlap each other, and therefore we have to consider short range effects such as an internal structure of hadrons, channel couplings to conventional three quark states and so on. As a naive criterion for the hadronic molecule, we have shown the relative radii

of the bound states as discussed in Ref [25]. If the size of the bound state is larger than twice of typical hadron size (namely $1 \mathrm{fm}$ ), the state could be well described by a molecular structure. For resonant states, we identify the states as the hadronic molecule. According to the criterion, for the $\pi \rho \omega$ potential, only the bound state for $J^{P}=3 / 2^{+}$of $\bar{B}^{(*)} N$ constructs a hadronic molecule, where the relative radius is $1.81 \mathrm{fm}$. Contrary to this, the bound states for $J^{P}=1 / 2^{ \pm}$and $3 / 2^{-}$with $I=0$ which have a small radius and a large binding energy are not described as simple molecules. We need to consider the short range effects including various channel couplings to do more realistic discussions.

\section{SUMMARY}

We have investigated heavy baryons as hadronic molecules formed by a heavy meson and a nucleon, $D^{(*)} N$ and $\bar{B}^{(*)} N$. The interaction is given by the meson exchange potential with respecting the heavy quark and chiral symmetries. Many bound states and resonances are 
found in isosinglet channel both for $D^{(*)} N$ and $\bar{B}^{(*)} N$ systems. In contrast, there are few resonances in isotriplet channel due to small isospin factor. For $J^{P}=1 / 2^{ \pm}$and $3 / 2^{-}$, we have found deeply bound states far below $D N$ or $\bar{B} N$ threshold with a small radius. In these states, the vector meson exchange potential yields a strong attraction. In order to perform more realistic discussions for such compact states, we need to consider further effects from internal structures of constituent hadrons and channel couplings to quark intrinsic states. On the other hand, for $J^{P}=3 / 2^{+}, 5 / 2^{ \pm}$and $7 / 2^{-}$with large orbital angular momenta, the one pion exchange potential is favored and we have obtained loosely bound states and resonances near the thresholds. There the short range interactions are rather inactive and the long range force of the one pion exchange dominates, where the tensor force plays an important role to generate these states, and they compose the hadronic molecular structure.

It is expected that the hadronic molecular states from a heavy meson and a nucleon are studied in many accelerator facilities; the $D$ meson production in the antiproton-nucleon annihilation process in FAIR [44] and the heavy ion collision in RHIC and LHC [45, 46]. Furthermore, the exotic baryons are investigated in J-PARC in the coming future.

\section{ACKNOWLEDGMENTS}

This work is supported in part by Grant-in-Aid for Scientific Research on Priority Areas "Elucidation of New Hadrons with a Variety of Flavors(E01: 21105006)" (S. Y. and A. H.) from the Ministry of Education, Culture, Sports, Science and Technology of Japan and Grant-in-Aid for "JSPS Fellows(24-3518)" (Y. Y.) from Japan Society for the Promotion of Science.

\section{Appendix A: Potentials and kinetic terms}

Interaction potentials are derived by using the Lagrangians in Eqs. (1), (2), (6) and (7)

as shown in Ref. [17, 18]. The potentials for the coupled channel systems are given in the matrix form of $3 \times 3$ for $J^{P}=1 / 2^{ \pm}$and of $4 \times 4$ for the other states. 
The $\pi$ exchange potentials for each $J^{P}$ are obtained by

$$
\begin{aligned}
& V_{1 / 2^{-}}^{\pi}=\left(\begin{array}{ccc}
0 & \sqrt{3} V_{C}^{\pi} & -\sqrt{6} V_{T}^{\pi} \\
\sqrt{3} V_{C}^{\pi} & -2 V_{C}^{\pi} & -\sqrt{2} V_{T}^{\pi} \\
-\sqrt{6} V_{T}^{\pi} & -\sqrt{2} V_{T}^{\pi} & V_{C}^{\pi}-2 V_{T}^{\pi}
\end{array}\right), \\
& V_{1 / 2^{+}}^{\pi}=\left(\begin{array}{ccc}
0 & \sqrt{3} V_{C}^{\pi} & -\sqrt{6} V_{T}^{\pi} \\
\sqrt{3} V_{C}^{\pi} & -2 V_{C}^{\pi} & -\sqrt{2} V_{T}^{\pi} \\
-\sqrt{6} V_{T}^{\pi} & -\sqrt{2} V_{T}^{\pi} & V_{C}^{\pi}-2 V_{T}^{\pi}
\end{array}\right), \\
& V_{3 / 2^{-}}^{\pi}=\left(\begin{array}{cccc}
0 & \sqrt{3} V_{T}^{\pi} & -\sqrt{3} V_{T}^{\pi} & \sqrt{3} V_{C}^{\pi} \\
\sqrt{3} V_{T}^{\pi} & V_{C}^{\pi} & 2 V_{T}^{\pi} & V_{T}^{\pi} \\
-\sqrt{3} V_{T}^{\pi} & 2 V_{T}^{\pi} & V_{C}^{\pi} & -V_{T}^{\pi} \\
\sqrt{3} V_{C}^{\pi} & V_{T}^{\pi} & -V_{T}^{\pi} & -2 V_{C}^{\pi}
\end{array}\right), \\
& V_{3 / 2^{+}}^{\pi}=\left(\begin{array}{cccc}
0 & \sqrt{3} V_{C}^{\pi} & \sqrt{\frac{3}{5}} V_{T}^{\pi} & -3 \sqrt{\frac{3}{5}} V_{T}^{\pi} \\
\sqrt{3} V_{C}^{\pi} & -2 V_{C}^{\pi} & \frac{1}{\sqrt{5}} V_{T}^{\pi} & -\sqrt{3} V_{T}^{\pi} \\
\sqrt{\frac{3}{5}} V_{T}^{\pi} & \frac{1}{\sqrt{5}} V_{T}^{\pi} & V_{C}^{\pi}+\frac{8}{5} V_{T}^{\pi} & \frac{6}{5} V_{T}^{\pi} \\
-3 \sqrt{\frac{3}{5}} V_{T}^{\pi} & -\sqrt{3} V_{T}^{\pi} & \frac{6}{5} V_{T}^{\pi} & V_{C}^{\pi}-\frac{8}{5} V_{T}^{\pi}
\end{array}\right), \\
& V_{5 / 2^{-}}^{\pi}=\left(\begin{array}{cccc}
0 & \sqrt{3} V_{C}^{\pi} & \sqrt{\frac{6}{7}} V_{T}^{\pi} & -\frac{6}{\sqrt{7}} V_{T}^{\pi} \\
\sqrt{3} V_{C}^{\pi} & -2 V_{C}^{\pi} & \sqrt{\frac{2}{7}} V_{T}^{\pi} & -2 \sqrt{\frac{3}{7}} V_{T}^{\pi} \\
\sqrt{\frac{6}{7}} V_{T}^{\pi} & \sqrt{\frac{2}{7}} V_{T}^{\pi} & V_{C}^{\pi}+\frac{10}{7} V_{T}^{\pi} & \frac{4}{7} \sqrt{6} V_{T}^{\pi} \\
-\frac{6}{\sqrt{7}} V_{T}^{\pi} & -2 \sqrt{\frac{3}{7}} V_{T}^{\pi} & \frac{4}{7} \sqrt{6} V_{T}^{\pi} & V_{C}^{\pi}-\frac{10}{7} V_{T}^{\pi}
\end{array}\right), \\
& V_{5 / 2^{+}}^{\pi}=\left(\begin{array}{cccc}
0 & \frac{3}{5} \sqrt{10} V_{T}^{\pi} & \sqrt{3} V_{C}^{\pi} & -2 \sqrt{\frac{3}{5}} V_{T}^{\pi} \\
\frac{3}{5} \sqrt{10} V_{T}^{\pi} & V_{C}^{\pi}-\frac{2}{5} V_{T}^{\pi} & \sqrt{\frac{6}{5}} V_{T}^{\pi} & \frac{4}{5} \sqrt{6} V_{T}^{\pi} \\
\sqrt{3} V_{C}^{\pi} & \sqrt{\frac{6}{5}} V_{T}^{\pi} & -2 V_{C}^{\pi} & -\frac{2}{\sqrt{5}} V_{T}^{\pi} \\
-2 \sqrt{\frac{3}{5}} V_{T}^{\pi} & \frac{4}{5} \sqrt{6} V_{T}^{\pi} & -\frac{2}{\sqrt{5}} V_{T}^{\pi} & V_{C}^{\pi}+\frac{2}{5} V_{T}^{\pi}
\end{array}\right),
\end{aligned}
$$




$$
\begin{gathered}
V_{7 / 2^{-}}^{\pi}=\left(\begin{array}{cccc}
0 & 3 \sqrt{\frac{3}{7}} V_{T}^{\pi} & \sqrt{3} V_{C}^{\pi} & -\sqrt{\frac{15}{7}} V_{T}^{\pi} \\
3 \sqrt{\frac{3}{7}} V_{T}^{\pi} & V_{C}^{\pi}-\frac{4}{7} V_{T}^{\pi} & \frac{3}{\sqrt{7}} V_{T}^{\pi} & \frac{6}{7} \sqrt{5} V_{T}^{\pi} \\
\sqrt{3} V_{C}^{\pi} & \frac{3}{\sqrt{7}} V_{T}^{\pi} & -2 V_{C}^{\pi} & -\sqrt{\frac{5}{7}} V_{T}^{\pi} \\
-\sqrt{\frac{15}{7}} V_{T}^{\pi} & \frac{6}{7} \sqrt{5} V_{T}^{\pi} & -\sqrt{\frac{5}{7}} V_{T}^{\pi} & V_{C}^{\pi}+\frac{4}{7} V_{T}^{\pi}
\end{array}\right), \\
V_{7 / 2^{+}}^{\pi}=\left(\begin{array}{cccc}
0 & \sqrt{3} V_{C}^{\pi} & V_{T}^{\pi} & -\sqrt{5} V_{T}^{\pi} \\
\sqrt{3} V_{C}^{\pi} & -2 V_{C}^{\pi} & \frac{1}{\sqrt{3}} V_{T}^{\pi} & -\sqrt{\frac{5}{3}} V_{T}^{\pi} \\
V_{T}^{\pi} & \frac{1}{\sqrt{3}} V_{T}^{\pi} & V_{C}^{\pi}+\frac{4}{3} V_{T}^{\pi} & \frac{2}{3} \sqrt{5} V_{T}^{\pi} \\
-\sqrt{5} V_{T}^{\pi} & -\sqrt{\frac{5}{3}} V_{T}^{\pi} & \frac{2}{3} \sqrt{5} V_{T}^{\pi} & V_{C}^{\pi}-\frac{4}{3} V_{T}^{\pi}
\end{array}\right),
\end{gathered}
$$

where

$$
V_{C}^{\pi}=\frac{g_{\pi} g_{\pi N N}}{\sqrt{2} m_{N} f_{\pi}} \frac{1}{3} C_{m_{\pi}} \vec{\tau}_{P} \cdot \vec{\tau}_{N}, \quad V_{T}^{\pi}=\frac{g_{\pi} g_{\pi N N}}{\sqrt{2} m_{N} f_{\pi}} \frac{1}{3} T_{m_{\pi}} \vec{\tau}_{P} \cdot \vec{\tau}_{N} .
$$

The $\vec{\tau}_{P}$ and $\vec{\tau}_{N}$ are the isospin matrices for $P^{(*)}$ and $N$. The functions $C_{m}=C(r ; m)$ and $T_{m}=T(r ; m)$ are given by

$$
\begin{aligned}
C(r ; m) & =\int \frac{d^{3} q}{(2 \pi)^{3}} \frac{m^{2}}{\vec{q}^{2}+m^{2}} e^{i \vec{q} \cdot \vec{r}} F\left(\Lambda_{P}, \vec{q}\right) F\left(\Lambda_{N}, \vec{q}\right), \\
T(r ; m) S_{12}(\hat{r}) & =\int \frac{d^{3} q}{(2 \pi)^{3}} \frac{-\vec{q}^{2}}{\vec{q}^{2}+m^{2}} S_{12}(\hat{q}) e^{i \vec{q} \cdot \vec{r}} F\left(\Lambda_{P}, \vec{q}\right) F\left(\Lambda_{N}, \vec{q}\right),
\end{aligned}
$$

with $S_{12}(\hat{x})=3\left(\vec{\sigma}_{1} \cdot \hat{x}\right)\left(\vec{\sigma}_{2} \cdot \hat{x}\right)-\vec{\sigma}_{1} \cdot \vec{\sigma}_{2}$, and $F(\Lambda, \vec{q})$ denotes the form factor given in Eq. (8).

The vector meson exchange potentials $(v=\rho, \omega)$ are

$$
\begin{aligned}
& V_{1 / 2^{-}}^{v}=\left(\begin{array}{ccc}
V_{C}^{v \prime} & 2 \sqrt{3} V_{C}^{v} & \sqrt{6} V_{T}^{v} \\
2 \sqrt{3} V_{C}^{v} & V_{C}^{v \prime}-4 V_{C}^{v} & \sqrt{2} V_{T}^{v} \\
\sqrt{6} V_{T}^{v} & \sqrt{2} V_{T}^{v} & V_{C}^{v \prime}+2 V_{C}^{v}+2 V_{T}^{v}
\end{array}\right), \\
& V_{1 / 2^{+}}^{v}=\left(\begin{array}{ccc}
V_{C}^{v \prime} & 2 \sqrt{3} V_{C}^{v} & \sqrt{6} V_{T}^{v} \\
2 \sqrt{3} V_{C}^{v} & V_{C}^{v \prime}-4 V_{C}^{v} & \sqrt{2} V_{T}^{v} \\
\sqrt{6} V_{T}^{v} & \sqrt{2} V_{T}^{v} & V_{C}^{v \prime}+2 V_{C}^{v}+2 V_{T}^{v}
\end{array}\right),
\end{aligned}
$$




$$
\begin{aligned}
& V_{3 / 2^{-}}^{v}=\left(\begin{array}{cccc}
V_{C}^{v \prime} & -\sqrt{3} V_{T}^{v} & \sqrt{3} V_{T}^{v} & 2 \sqrt{3} V_{C}^{v} \\
-\sqrt{3} V_{T}^{v} & V_{C}^{v \prime}+2 V_{C}^{v} & -2 V_{T}^{v} & -V_{T}^{v} \\
\sqrt{3} V_{T}^{v} & -2 V_{T}^{v} & V_{C}^{v \prime}+2 V_{C}^{v} & V_{T}^{v} \\
2 \sqrt{3} V_{C}^{v} & -V_{T}^{v} & V_{T}^{v} & V_{C}^{v \prime}-4 V_{C}^{v}
\end{array}\right), \\
& V_{3 / 2^{+}}^{v}=\left(\begin{array}{cccc}
V_{C}^{v \prime} & 2 \sqrt{3} V_{C}^{v} & -\sqrt{\frac{3}{5}} V_{T}^{v} & 3 \sqrt{\frac{3}{5}} V_{T}^{v} \\
2 \sqrt{3} V_{C}^{v} & V_{C}^{v \prime}-4 V_{C}^{v} & -\frac{1}{\sqrt{5}} V_{T}^{v} & \frac{3}{\sqrt{5}} V_{T}^{v} \\
-\sqrt{\frac{3}{5}} V_{T}^{v} & -\frac{1}{\sqrt{5}} V_{T}^{v} & V_{C}^{v \prime}+2 V_{C}^{v}-\frac{8}{5} V_{T}^{v} & -\frac{6}{5} V_{T}^{v} \\
3 \sqrt{\frac{3}{5}} V_{T}^{v} & \frac{3}{\sqrt{5}} V_{T}^{v} & -\frac{6}{5} V_{T}^{v} & V_{C}^{v \prime}+2 V_{C}^{v}+\frac{8}{5} V_{T}^{v}
\end{array}\right), \\
& V_{5 / 2^{-}}^{v}=\left(\begin{array}{cccc}
V_{C}^{v \prime} & 2 \sqrt{3} V_{C}^{v} & -\sqrt{\frac{6}{7}} V_{T}^{v} & \frac{6}{\sqrt{7}} V_{T}^{v} \\
2 \sqrt{3} V_{C}^{v} & V_{C}^{v \prime}-4 V_{C}^{v} & -\sqrt{\frac{2}{7}} V_{T}^{v} & 2 \sqrt{\frac{3}{7}} V_{T}^{v} \\
-\sqrt{\frac{6}{7}} V_{T}^{v} & -\sqrt{\frac{2}{7}} V_{T}^{v} & V_{C}^{v \prime}+2 V_{C}^{v}-\frac{10}{7} V_{T}^{v} & -\frac{4}{7} \sqrt{6} V_{T}^{v} \\
\frac{6}{\sqrt{7}} V_{T}^{v} & 2 \sqrt{\frac{3}{7}} V_{T}^{v} & -\frac{4}{7} \sqrt{6} V_{T}^{v} & V_{C}^{v \prime}+2 V_{C}^{v}+\frac{10}{7} V_{T}^{v}
\end{array}\right), \\
& V_{5 / 2^{+}}^{v}=\left(\begin{array}{cccc}
V_{C}^{v \prime} & -\frac{3}{5} \sqrt{10} V_{T}^{v} & 2 \sqrt{3} V_{C}^{v} & 2 \sqrt{\frac{3}{5}} V_{T}^{v} \\
-\frac{3}{5} \sqrt{10} V_{T}^{v} & V_{C}^{v \prime}+2 V_{C}^{v}+\frac{2}{5} V_{T}^{v} & -\sqrt{\frac{6}{5}} V_{T}^{v} & -\frac{4}{5} \sqrt{6} V_{T}^{v} \\
2 \sqrt{3} V_{C}^{v} & -\sqrt{\frac{6}{5}} V_{T}^{v} & V_{C}^{v \prime}-4 V_{C}^{v} & \frac{2}{\sqrt{5}} V_{T}^{v} \\
2 \sqrt{\frac{3}{5}} V_{T}^{v} & -\frac{4}{5} \sqrt{6} V_{T}^{v} & \frac{2}{\sqrt{5}} V_{T}^{v} & V_{C}^{v \prime}+2 V_{C}^{v}-\frac{2}{5} V_{T}^{v}
\end{array}\right), \\
& V_{7 / 2^{-}}^{v}=\left(\begin{array}{cccc}
V_{C}^{v \prime} & -3 \sqrt{\frac{3}{7}} V_{T}^{v} & 2 \sqrt{3} V_{C}^{v} & \sqrt{\frac{15}{7}} V_{T}^{v} \\
-3 \sqrt{\frac{3}{7}} V_{T}^{v} & V_{C}^{v \prime}+2 V_{C}^{v}+\frac{4}{7} V_{T}^{v} & -\frac{3}{\sqrt{7}} V_{T}^{v} & -\frac{6}{7} \sqrt{5} V_{T}^{v} \\
2 \sqrt{3} V_{C}^{v} & -\frac{3}{\sqrt{7}} V_{T}^{v} & V_{C}^{v \prime}-4 V_{C}^{v} & \sqrt{\frac{5}{7}} V_{T}^{v} \\
\sqrt{\frac{15}{7}} V_{T}^{v} & -\frac{6}{7} \sqrt{5} V_{T}^{v} & \sqrt{\frac{5}{7}} V_{T}^{v} & V_{C}^{v \prime}+2 V_{C}^{v}-\frac{4}{7} V_{T}^{v}
\end{array}\right),
\end{aligned}
$$




$$
V_{7 / 2^{+}}^{v}=\left(\begin{array}{cccc}
V_{C}^{v \prime} & 2 \sqrt{3} V_{C}^{v} & -V_{T}^{v} & \sqrt{5} V_{T}^{v} \\
2 \sqrt{3} V_{C}^{v} & V_{C}^{v \prime}-4 V_{C}^{v} & -\frac{1}{\sqrt{3}} V_{T}^{v} & \sqrt{\frac{5}{3}} V_{T}^{v} \\
-V_{T}^{v} & -\frac{1}{\sqrt{3}} V_{T}^{v} & V_{C}^{v \prime}+2 V_{C}^{v}-\frac{4}{3} V_{T}^{v} & -\frac{2}{3} \sqrt{5} V_{T}^{v} \\
\sqrt{5} V_{T}^{v} & \sqrt{\frac{5}{3}} V_{T}^{v} & -\frac{2}{3} \sqrt{5} V_{T}^{v} & V_{C}^{v \prime}+2 V_{C}^{v}+\frac{4}{3} V_{T}^{v}
\end{array}\right),
$$

where $V_{C}^{v \prime}, V_{C}^{v}$ and $V_{T}^{v}$ are defined as

$$
\begin{aligned}
V_{C}^{\rho \prime} & =\frac{g_{V} g_{\rho N N} \beta}{\sqrt{2} m_{\rho}^{2}} C_{m_{\rho}} \vec{\tau}_{P} \cdot \vec{\tau}_{N}, \\
V_{C}^{\rho} & =\frac{g_{V} g_{\rho N N} \lambda(1+\kappa)}{\sqrt{2} m_{N}} \frac{1}{3} C_{m_{\rho}} \vec{\tau}_{P} \cdot \vec{\tau}_{N}, \\
V_{T}^{\rho} & =\frac{g_{V} g_{\rho N N} \lambda(1+\kappa)}{\sqrt{2} m_{N}} \frac{1}{3} T_{m_{\rho}} \vec{\tau}_{P} \cdot \vec{\tau}_{N}, \\
V_{C}^{\omega \prime} & =\frac{g_{V} g_{\omega N N} \beta}{\sqrt{2} m_{\omega}^{2}} C_{m_{\omega}}, \\
V_{C}^{\omega} & =\frac{g_{V} g_{\omega N N} \lambda}{\sqrt{2} m_{N}} \frac{1}{3} C_{m_{\omega}}, \\
V_{T}^{\omega} & =\frac{g_{V} g_{\omega N N} \lambda}{\sqrt{2} m_{N}} \frac{1}{3} T_{m_{\omega}} .
\end{aligned}
$$

Finally, the kinetic terms are given by

$$
\begin{aligned}
& K_{1 / 2^{-}}=\operatorname{diag}(\left.-\frac{1}{2 \tilde{m}_{P}} \triangle_{0},-\frac{1}{2 \tilde{m}_{P^{*}}} \triangle_{0}+\Delta m_{P P^{*}},-\frac{1}{2 \tilde{m}_{P^{*}}} \triangle_{2}+\Delta m_{P P^{*}}\right), \\
& K_{1 / 2^{+}}=\operatorname{diag}\left(-\frac{1}{2 \tilde{m}_{P}} \triangle_{1},-\frac{1}{2 \tilde{m}_{P^{*}}} \triangle_{1}+\Delta m_{P P^{*}},-\frac{1}{2 \tilde{m}_{P^{*}}} \triangle_{1}+\Delta m_{P P^{*}}\right), \\
& K_{3 / 2^{-}}=\operatorname{diag}\left(-\frac{1}{2 \tilde{m}_{P}} \triangle_{2},-\frac{1}{2 \tilde{m}_{P^{*}}} \triangle_{0}+\Delta m_{P P^{*}},-\frac{1}{2 \tilde{m}_{P^{*}}} \triangle_{2}+\Delta m_{P P^{*}},\right. \\
&\left.-\frac{1}{2 \tilde{m}_{P^{*}}} \triangle_{2}+\Delta m_{P P^{*}}\right), \\
& K_{3 / 2^{+}}= \operatorname{diag}\left(-\frac{1}{2 \tilde{m}_{P}} \triangle_{1},-\frac{1}{2 \tilde{m}_{P^{*}}} \triangle_{1}+\Delta m_{P P^{*}},-\frac{1}{2 \tilde{m}_{P^{*}}} \triangle_{1}+\Delta m_{P P^{*}},\right. \\
&\left.-\frac{1}{2 \tilde{m}_{P^{*}}} \triangle_{3}+\Delta m_{P P^{*}}\right),
\end{aligned}
$$




$$
\begin{aligned}
K_{5 / 2^{-}}= & \operatorname{diag}\left(-\frac{1}{2 \tilde{m}_{P}} \triangle_{2},-\frac{1}{2 \tilde{m}_{P^{*}}} \triangle_{2}+\Delta m_{P P^{*}},-\frac{1}{2 \tilde{m}_{P^{*}}} \triangle_{2}+\Delta m_{P P^{*}},\right. \\
& \left.-\frac{1}{2 \tilde{m}_{P^{*}}} \triangle_{4}+\Delta m_{P P^{*}}\right) \\
K_{5 / 2^{+}}= & \operatorname{diag}\left(-\frac{1}{2 \tilde{m}_{P}} \triangle_{3},-\frac{1}{2 \tilde{m}_{P^{*}}} \triangle_{1}+\Delta m_{P P^{*}},-\frac{1}{2 \tilde{m}_{P^{*}}} \triangle_{3}+\Delta m_{P P^{*}},\right. \\
& \left.-\frac{1}{2 \tilde{m}_{P^{*}}} \triangle_{3}+\Delta m_{P P^{*}}\right), \\
K_{7 / 2^{-}}= & \operatorname{diag}\left(-\frac{1}{2 \tilde{m}_{P}} \triangle_{4},-\frac{1}{2 \tilde{m}_{P^{*}}} \triangle_{2}+\Delta m_{P P^{*}},-\frac{1}{2 \tilde{m}_{P^{*}}} \triangle_{4}+\Delta m_{P P^{*}},\right. \\
& \left.-\frac{1}{2 \tilde{m}_{P^{*}}} \triangle_{4}+\Delta m_{P P^{*}}\right), \\
K_{7 / 2^{+}=} & \operatorname{diag}\left(-\frac{1}{2 \tilde{m}_{P}} \triangle_{3},-\frac{1}{2 \tilde{m}_{P^{*}}} \triangle_{3}+\Delta m_{P P^{*}},-\frac{1}{2 \tilde{m}_{P^{*}}} \triangle_{3}+\Delta m_{P P^{*}},\right. \\
& \left.-\frac{1}{2 \tilde{m}_{P^{*}}} \triangle_{5}+\Delta m_{P P^{*}}\right),
\end{aligned}
$$

where $\triangle_{l}=\partial^{2} / \partial r^{2}+(2 / r) \partial / \partial r-l(l+1) / r^{2}, \tilde{m}_{P^{(*)}}=m_{N} m_{P^{(*)}} /\left(m_{N}+m_{P^{(*)}}\right)$, and $\Delta m_{P P^{*}}=$ $m_{P^{*}}-m_{P}$. The total Hamiltonian is then given by $H_{J^{P}}=K_{J^{P}}+V_{J^{P}}$.

[1] S. K. Choi et al. [Belle Collaboration], Phys. Rev. Lett. 91 (2003) 262001 hep-ex/0309032].

[2] M. B. Voloshin, Prog. Part. Nucl. Phys. 61 (2008) 455 arXiv:0711.4556 [hep-ph]].

[3] E. S. Swanson, Phys. Rept. 429 (2006) 243 hep-ph/0601110.

[4] N. Brambilla, S. Eidelman, B. K. Heltsley, R. Vogt, G. T. Bodwin, E. Eichten, A. D. Frawley and A. B. Meyer et al., Eur. Phys. J. C 71 (2011) 1534 [arXiv:1010.5827 [hep-ph]].

[5] A. Bondar et al. [Belle Collaboration], Phys. Rev. Lett. 108 (2012) 122001 arXiv:1110.2251 [hep-ex]].

[6] I. Adachi et al. [Belle Collaboration], arXiv:1207.4345 [hep-ex].

[7] M. B. Voloshin, Phys. Rev. D 84 (2011) 031502 [arXiv:1105.5829 [hep-ph]].

[8] A. E. Bondar, A. Garmash, A. I. Milstein, R. Mizuk and M. B. Voloshin, Phys. Rev. D 84 (2011) 054010 arXiv:1105.4473 [hep-ph]].

[9] See for example Ref. [10] for recent review for charmed and bottom baryons as well as up, down, strangeness baryons. 
[10] E. Klempt and J. -M. Richard, Rev. Mod. Phys. 82, 1095 (2010) arXiv:0901.2055 [hep-ph]].

[11] L. A. Copley, N. Isgur and G. Karl, Phys. Rev. D 20, 768 (1979) [Erratum-ibid. D 23, 817 (1981)].

[12] W. Roberts and M. Pervin, Int. J. Mod. Phys. A 23 (2008) 2817 arXiv:0711.2492 [nucl-th]].

[13] N. Isgur and M. B. Wise, Phys. Lett. B 232 (1989) 113.

[14] N. Isgur and M. B. Wise, Phys. Rev. Lett. 66 (1991) 1130.

[15] The notation $P$ in the present article is opposite in charge conjugation from those in Refs. [1618].

[16] S. Yasui and K. Sudoh, Phys. Rev. D 80 (2009) 034008 arXiv:0906.1452 [hep-ph]].

[17] Y. Yamaguchi, S. Ohkoda, S. Yasui and A. Hosaka, Phys. Rev. D 84 (2011) 014032 arXiv:1105.0734 [hep-ph]].

[18] Y. Yamaguchi, S. Ohkoda, S. Yasui and A. Hosaka, Phys. Rev. D 85 (2012) 054003 arXiv:1111.2691 [hep-ph]].

[19] K. Ikeda, T. Myo, K. Kato and H. Toki, Lect. Notes Phys. 818, 165 (2010) arXiv:1007.2474 [nucl-th]].

[20] N. Kaiser, R. Brockmann and W. Weise, Nucl. Phys. A 625 (1997) 758 nucl-th/9706045.

[21] N. Kaiser, S. Gerstendorfer and W. Weise, Nucl. Phys. A 637 (1998) 395 nucl-th/9802071.

[22] R. J. N. Phillips, Rev. Mod. Phys. 39 (1967) 681.

[23] C. B. Dover and J. M. Richard, Phys. Rev. D 17 (1978) 1770.

[24] S. Ohkoda, Y. Yamaguchi, S. Yasui, K. Sudoh and A. Hosaka, Phys. Rev. D 86 (2012) 014004 arXiv:1111.2921 [hep-ph]].

[25] S. Ohkoda, Y. Yamaguchi, S. Yasui, K. Sudoh and A. Hosaka, arXiv:1202.0760 [hep-ph].

[26] T. Mizutani and A. Ramos, Phys. Rev. C 74 (2006) 065201 hep-ph/0607257.

[27] C. Garcia-Recio, V. K. Magas, T. Mizutani, J. Nieves, A. Ramos, L. L. Salcedo and L. Tolos, Phys. Rev. D 79 (2009) 054004 arXiv:0807.2969 [hep-ph]].

[28] O. Romanets, L. Tolos, C. Garcia-Recio, J. Nieves, L. L. Salcedo and R. G. E. Timmermans, Phys. Rev. D 85 (2012) 114032 [arXiv:1202.2239 [hep-ph]].

[29] C. Garcia-Recio, J. Nieves, O. Romanets, L. L. Salcedo and L. Tolos, arXiv:1210.4755 [hep-ph].

[30] J. Hofmann and M. F. M. Lutz, Nucl. Phys. A 763, 90 (2005) hep-ph/0507071.

[31] J. Hofmann and M. F. M. Lutz, Nucl. Phys. A 776, 17 (2006) hep-ph/0601249.

[32] J. Haidenbauer, G. Krein, U. -G. Meissner and L. Tolos, Eur. Phys. J. A 47 (2011) 18 
arXiv:1008.3794 [nucl-th]].

[33] J. He, and X. Liu, Phys. Rev. D 82, 114029 (2010) arXiv:1008.1500 [hep-ph]].

[34] J. -R. Zhang, arXiv:1211.2277 [hep-ph].

[35] J. -R. Zhang, arXiv:1212.5325 [hep-ph].

[36] J. -J. Wu, R. Molina, E. Oset and B. S. Zou, Phys. Rev. Lett. 105 (2010) 232001 arXiv:1007.0573 [nucl-th]].

[37] J. -J. Wu, R. Molina, E. Oset and B. S. Zou, Phys. Rev. C 84 (2011) 015202 [arXiv:1011.2399 [nucl-th]].

[38] J. -J. Wu and B. S. Zou, Phys. Lett. B 709 (2012) 70 [arXiv:1011.5743 [hep-ph]].

[39] A. V. Manohar and M. B. Wise, Camb. Monogr. Part. Phys. Nucl. Phys. Cosmol. 10, 1-191 (2000).

[40] R. Casalbuoni, A. Deandrea, N. Di Bartolomeo, R. Gatto, F. Feruglio and G. Nardulli, Phys. Rept. 281 (1997) 145 arXiv:hep-ph/9605342.

[41] M. Bando, T. Kugo and K. Yamawaki, Phys. Rept. 164 (1988) 217.

[42] R. Machleidt, K. Holinde and C. Elster, Phys. Rept. 149 (1987) 1; R. Machleidt, Phys. Rev. C 63 (2001) 024001 arXiv:nucl-th/0006014.

[43] K. Arai and A. T. Kruppa, Phys. Rev. C 60 (1999) 064315.

[44] U. Wiedner, Prog. Part. Nucl. Phys. 66 (2011) 477 [arXiv:1104.3961 [hep-ex]].

[45] S. Cho et al. [ExHIC Collaboration], Phys. Rev. Lett. 106 (2011) 212001 arXiv:1011.0852 [nucl-th]].

[46] S. Cho et al. [ExHIC Collaboration], Phys. Rev. C 84 (2011) 064910 arXiv:1107.1302 [nuclth]]. 